\title{
Design of Data Display Based on the Food Safety
}

\section{Traceability}

\author{
Yu qunxiu \\ College of Information Engineering and Automation \\ Kunming University of Science and Technology \\ Kunming, Yunnan, China \\ E-mail: qingdaoyqx@163.com \\ Zhang shouming* \\ College of Information Engineering and Automation \\ Kunming University of Science and Technology \\ Kunming, Yunnan, China E-mail: \\ qingdaoyqx@163.com \\ * Corresponding Author
}

\author{
Zhang heng \\ College of Information Engineering and Automation \\ Kunming University of Science and Technology \\ Kunming, Yunnan, China \\ E-mail: 489638065@qq.com \\ Wang chao \\ College of Information Engineering and Automation \\ Kunming University of Science and Technology \\ Kunming, Yunnan, China \\ E-mail: wangchao19890516@163.com
}

\begin{abstract}
In view of the test about the design of food safety traceability control platform, this paper proposes a data to display detection method, It uses a more intuitive LED display and speech broadcast module to display and report food safety traceability information, the basic principle of this design is that when consumers credit card, electronic tag number receives date via Zigbee receiving module, under the control of the microcontroller reads the corresponding electronic tag number corresponding food safety information and for display on LED display, at the same time for voice module of speech. The system has low power consumption, strong anti-jamming capability, good general type, the advantages of circuit is simple and reliable and has small volume. At the same time, by the microcontroller instead of PC to control its function module, not only can save cost, also can make the system simpler. Experimental results show that this method is not only suitable for life and easy to carry out supervision and inspection departments, but also facilitate the supervision of consumers buying food safety.

Keywords-data displa; LED displa; voice broadcast; Zigbee data; safe
\end{abstract}

\section{INTRODUCTION}

The current food safety problems frequently occur, seriously harm people's health, so as to solve the problem of food safety has become the primary issue in the field of food ${ }^{[1]}$. This topic mainly regard pork products as the research object, in view of the pork market develop a set of food safety traceability control platforms, using LED display and speech function of pork food safety information for effective management and control. Food safety traceability control platform based on the food safety information for storage, after the brushing electronic tags will be showed food safety traceability information through the LED display, voice broadcast multiple platforms to show, not only let consumers more intuitive and convenient to buy food to the corresponding information. At the same time, letting the LED display, speech technology applied to our ordinary people's life, making the development of these technologies get more fully. This paper is arranged as follows: section 2 introduces system overall scheme design; Section 3 introduces the LED display module design; Section 4 introduces speech module design; Section 5 experimental conclusions; finally summarizes for this article.

\section{THE SYSTEM OF OVERALL SCHEME DESIGN}

As food safety information tracing control platform of terminal market revelation of major food safety information, and meet consumers' right to know and option for food, strengthen the confidence of the buyer ${ }^{[2]}$. Market in every piece of pork with electronic label, and in the database has corresponded electronic tag pig food safety information, so the market terminal information of food safety traceability control platform need to receive and storage, food safety information on electronic tag number for collection, electronic tag number corresponding to the food safety information for LED display and speech broadcast, etc.

The source of the food safety information is formed after slaughter inspection qualified, through GPRS sent to various stalls, market terminal consumers only need to brush the electronic label can see the corresponding information of pork. To solve the security problems of "from the source to the table ${ }^{[3] " .}$

This design uses the GPRS Modem for data wireless receive and use SD card to store the received food safety information, using the card reader to realize the collection of electronic tag number, using the Zigbee to realize electronic tag data wireless transmission, food safety information to make use of the LED display, at the same time equipped with speech module for food safety information, implementation of food safety information completely transparent. The corresponding topological structure models are shown in Fig .1: 


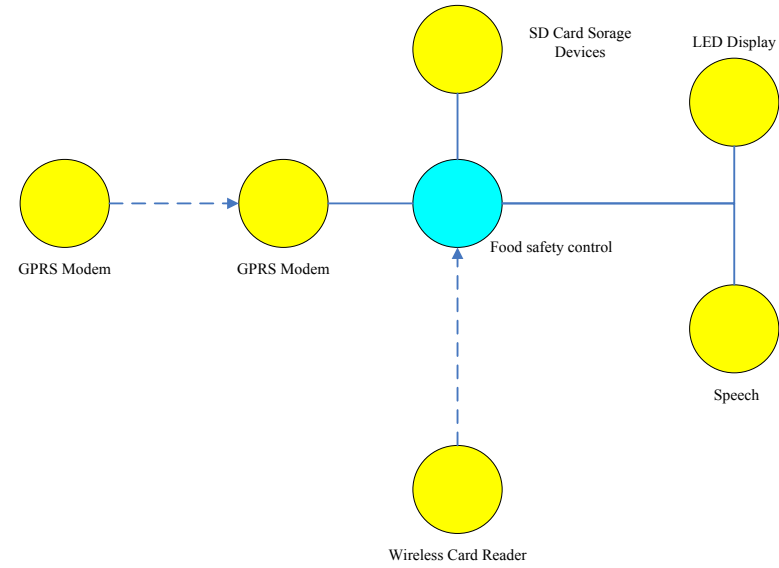

Figure 1. System overall scheme design

Considered in the design of funds and function can be realized, the control sections are achieved by the microcontroller ${ }^{[4]}$. First to receive food safety information through GPRS Modem, through SCM and SD card module communicate food safety information into the SD card $^{[5]}$; When consumers need to know the safety information of the product they want to buy, the use of wireless electronic tag reader reads the electronic brush tag number, and transmitted via Zigbee electronic tag number to the master controller; SD card host controller reads the stored file name (name of food electronic tag number) and the main control terminal Zigbee received electronic tag number comparison, find the corresponding file when the file is passed to the data read LED and voice module and voice broadcast for display, and for the food display complete information from breeding to sell to consumers. To this end the following functional block diagram of the overall design of the system is shown in Fig .2.

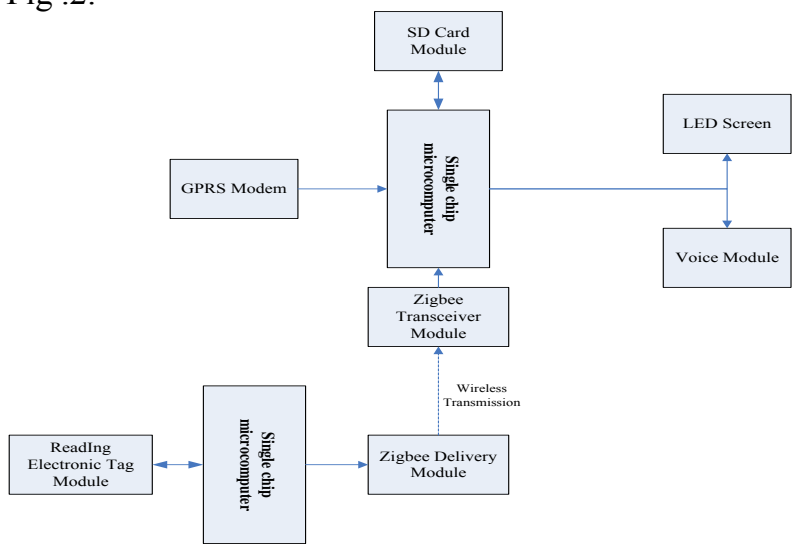

Figure 2. The function of the system structure diagram

Today in the rapid development of science and technology, all of the application include RFID technology, Zigbee technology, single-chip microcomputer technology, the LED display technology, speech technology, u disk read and write technology are not significant technical problems, the system is the organic integration of these and become a complete system. Therefore, the system is feasible in technology.

\section{THE KEY TECHNOLOGY}

Food safety information display module mainly consists of six parts, respectively is: single chip microcomputer, Zigbee data receiving module and GPRS Modem SMS receiving module, the SD card data storage module, LED display module and speech module. GPRS Modem receives the food safety information in the SD card for the call, when consumer credit card number through the Zigbee data receiving module, under the control of the microcontroller reads the corresponding electronic tag number corresponding to the food safety information and LED display on the screen, at the same time speech voice modules. To design a food safety information display module of hardware function are shown in Fig .3.

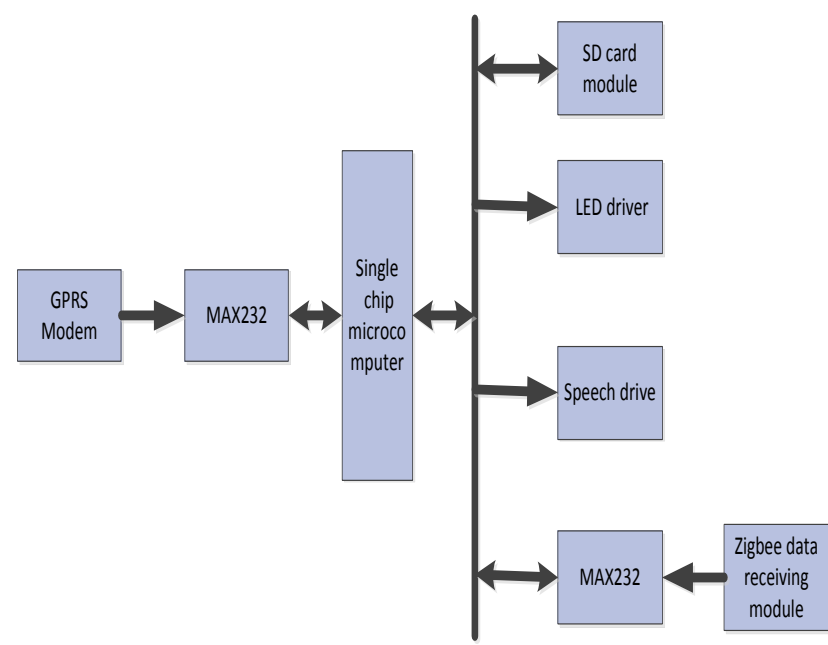

Figure 3. The hardware block diagram of information display module

\section{A. Single chip microcomputer technology}

SCM is also called the single chip microcomputer, is a typical micro controller, it is a microcomputer ${ }^{[6]}$ and integrated by the CPU, registers, RAM/ROM, I/O interface circuit and so on integrated on a chip. Microcontroller is composed of two parts of hardware and software, hardware consists of memory, input and output devices, such as CPU, the software is composed of a variety of applications. There are many kinds of single chip microcomputer and according to the purpose can be divided into two categories: special or not special (universal), special is developed for a particular area of equipment, universal criterion can be applied in various industries; In accordance with the figures can be divided into $4,8,16$ and 32 categories, eight microcontroller is used widely.

At present, the MCS - 51 single chip microcomputer is the most widely used domestic an 8-bit microcontroller, it has small volume, low power consumption and high cost performance, strong anti-interference ability, high reliability, wide application range, and many other advantages, in the industrial control, sophisticated weapons, communications equipment, information processing, household appliances and other fields of application.

\section{B. RFID technology}


RFID (Radio Frequency Identification, RFID for short), the Radio Frequency Identification (RFID), commonly known as electronic tag ${ }^{[7]}$. It is an advanced non-contact automatic identification technology, it through radio frequency signal automatically identify target objects and access to relevant data, identify work without manual intervention, it can work in all kinds of bad environment. RFID technology can identify the highspeed moving objects and can identify multiple tags at the same time, fast and convenient operation. Commonly used in meat food quality and safety traceability management, because of its recognition speed, high accuracy, is conducive to improve the efficiency of information collection; Label strong pollution resistance, high and low temperature resistance, durability, suitable for meat processing and sales of application environment; Large amount of information storage capabilities, meet the meat the needs of each link in the supply chain security information record transfer. RFID system compared with traditional dimensional barcode, the recognition rate is high, the characteristics of non-contact scanning, and a larger volume, will further improve the efficiency of "traceability" of food, and improve the quality of food safety.

RFID is made up of three parts, label (Tag), Reader (Reader) and Antenna (can Antenna) to be used. Label (Tag) is composed of coupling components and chips, each Tag has a unique electronic coding, attached to the object identified target; (Reader) is the equipment label to read (write), can design for a handheld or stationary; Antenna (can Antenna) to be used to send signal transmission between tag and reader.

The working principle of RFID technology: the Tag into the magnetic field, after receiving rf signal from the reader, with the energy gained by the induced current send product information stored in the chip (Passive Tag, Passive tags or Passive tags), or by the label initiative to send a frequency signal (Active Tag, Active tags or Active tags), the reader information and decoding, sent to the central information system on data processing, need other hardware and software in the practical application support $^{[8]}$

\section{Zigbee technology}

Zigbee technology is a kind of close, low complexity and low cost, low power consumption, low rate of twoway wireless communication technology. Is mainly used for short distance and low power consumption and transmission rate is not high to data transmission between various kinds of electronic equipment, as well as the typical periodic data, intermittent data and low response time data transmission applications ${ }^{[9]}$. It combines sensor technology, communication technology, control technology, many technologies such as its wide application of improved people's quality of life greatly, at the same time also need small amount of data and provides a new technology for the industrial application.

\section{LED DISPLAY MODULE DESIGN}

LED is used in the design of the display $128 * 64,32$ points a character, a total of four lines, each line can display 8 characters. Since the LED without a character, in order to facilitate the operation, design ready Chinese characters used in conjunction with the card. As described in the hardware design, using serial communication with the microcontroller, the microcontroller via a serial port to send commands and data to control the LED display content and manner. Which control commands are: start playing, stop playing, restarting, clear the screen, show text immediately and so on. Each control command format is as follows.

TABLE I. Control command format

\begin{tabular}{|c|c|c|}
\hline $\begin{array}{l}\text { Ordered } \\
\text { item }\end{array}$ & $\begin{array}{l}\text { Comman } \\
\text { d code }\end{array}$ & Instructions \\
\hline Start playing & $\begin{array}{l}5 \mathrm{~A} 0193 \\
0000 \text { A5 }\end{array}$ & $\begin{array}{c}\text { 5ASynchronous } \\
\text { character,01screen,93/90/91/ }\end{array}$ \\
\hline Stop playing & $\begin{array}{l}5 \mathrm{~A} 0190 \\
0000 \mathrm{~A} 5\end{array}$ & $\begin{array}{l}81 \text { order, } 00 \text { length of the } \\
\text { data,00 A5 The end of the }\end{array}$ \\
\hline Restart & $\begin{array}{l}5 \mathrm{~A} 0191 \\
0000 \text { A5 }\end{array}$ & character \\
\hline $\begin{array}{l}\text { Clear the } \\
\text { screen }\end{array}$ & $\begin{array}{l}5 \mathrm{~A} 0181 \\
0000 \mathrm{~A} 5\end{array}$ & \\
\hline $\begin{array}{l}\text { Immediately } \\
\text { show }\end{array}$ & $\begin{array}{ccc}5 \mathrm{~A} & 0185 \\
\text { 0A } 2810 \\
\text { B0 A1 } 30 \\
\text { F7 FE } 41 \\
\text { A1 E6 } 00 \\
\text { A5 }\end{array}$ & $\begin{array}{l}\text { 5ASynchronous character,01 } \\
\text { screen, } 85 \text { order,0Aength of } \\
\text { the data (Max240), } 28 \\
\text { Starting point } \mathrm{X} \\
\text { coordinate, } 10 \text { Starting point } \\
\text { Y coordinate, other data is } \\
\text { GB2312 coding, } 00 \text { A5 The } \\
\text { end of the character }\end{array}$ \\
\hline
\end{tabular}

Static display in LED design, first sends a command to stop playing, then clear the screen, finally were in the first row, second row, third line, fourth line shows the static content. Corresponding program flow chart shown in Fig.4.

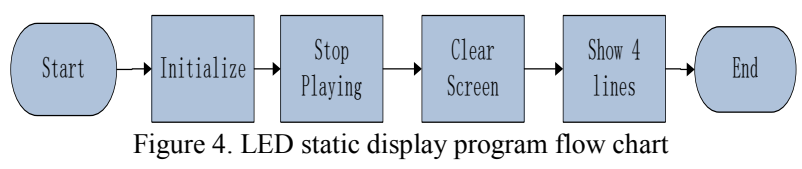

When the microcontroller writes data to each line LED display, first send control commands, and then send the data, and finally end the command needs to be sent, in order to achieve a complete LED display content specific flow chart as shown in Fig .5:

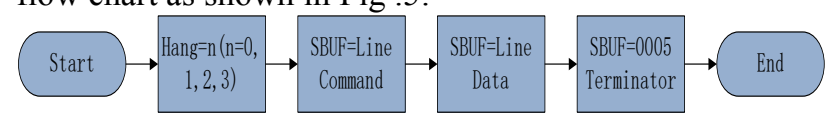

Figure 5. LED static display program flow chart

\section{SPEECH MODULE DESIGN}

In the design of this speech choose hkust xunfei XF S4240 special speech synthesis module ${ }^{[10]}$, the design of 
single chip microcomputer as the host, the XF - S4240 special speech synthesis module, as from the machine, and XF - address $0 \times 80$ S4240. IIC bus communication needs to start signal, the response signals, stop, start and stop signals are made by the host, after the starting signal, the bus is in a state of being used; After the termination of signal, the bus is idle. Each byte must ensure that the length is eight. When data transmission, transfer first highest (MSB), each byte transferred must follow a response (i.e., a frame with a total of nine). After the start signal must be sent from a machine address (7), the eighth is the data transfer direction $(\mathrm{R} / \mathrm{T})$, expressed as " 0 " the host to send data (T), "1" said host receives data (R). Each data transfer is always end termination signal produced by the host. But, if the host wants to continue to take bus to new data transfer, you cannot produce termination signal, again send out immediately start signal for addressing from another machine.

His design USES the host to send data from the machine, data transfer direction in the whole process of transmitting the same form, format as follows:

\begin{tabular}{c|c|l|l|l|l|l|l|l|}
\hline S & machine address & 0 & A & DATA & A & DATA & A $/ A$ & P \\
\hline
\end{tabular}

Figure 6. Direction of data transfer

Note: there are shaded part said data transmitted from the host to the machine, no shadow part said data from the machine to the host. A for the reply, not A said Not response (high level). $S$ said start signal, $P$ said termination signal.

In the realization of speech synthesis ${ }^{[11]}$, single-chip microcomputer sends a command to the XF - S4240 first send start signal at address 0 x80 choose XF - S4240 as from the machine, after waiting for the XF - S4240 response, send voice synthesis command, the command format as follows:

\begin{tabular}{|c|c|c|c|c|c|}
\hline Frame Header & \multicolumn{2}{|c|}{ Lenggth Of Data Area } & \multicolumn{3}{|c|}{ Data Area } \\
\hline \multirow{2}{*}{ OxFD } & High Byte & Low Byte & $\begin{array}{c}\text { Command } \\
\text { nord }\end{array}$ & Text encoding format & $\begin{array}{c}\text { Waiting ș̣nthesis } \\
\text { text }\end{array}$ \\
\hline & $\mathrm{OxHH}$ & OxLL & $\mathrm{OrOl}$ & $\mathrm{Ox} 01 \sim 0 \mathrm{x} 03$ & .'.'.' \\
\hline
\end{tabular}

Figure 7. The command format

Among them, each sending a byte of data to wait for the XF - S4240 reply, stay after completion of all the data sent, microcontroller send the stop signal, termination of synthesis. The corresponding program flow charts are shown in Fig .8:

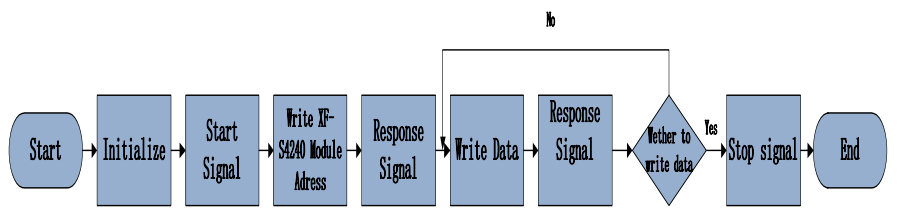

Figure 8. speech broadcast program flow chart

\section{CONCLUSION}

Experiments prove that food safety traceability control platform, data display design adopted the general electronic components, using single-chip microcomputer and peripheral interface to realize the LED display show and speech broadcast module, the software and hardware organically, enables the system to correctly display of food traceability data information and reporting, so as to realize the function of design. First is LED display, it has prominent characteristics, through the single chip microcomputer program can display the corresponding Chinese characters, is we to the naked eye can observe. Finally, if speech broadcast module the work is normal, can the corresponding sound.

\section{REFERENCES}

[1] M FALLON. Traceability of poultry and poultry products. Rev sci tech of int EPiz, 2001, 20(2) : 538-546.

[2] LU Q, LU J, HUANG B, et a1. Establishment and application of food defame plan in food processing enterprises. Asian Agricultural Research, 2009, 1(2): 21-23, 28

[3] Zhao jinyan, Tao linli, Gao shizheng, Ge changrong, zhang xi. Research on the animal food safety can be traced back system based on RFID technology research [journal]. Journal of yunnan agricultural university, 2008 (7) : 23 (4).

[4] Xiao kan, Li qunfang. Single chip microcomputer principle and application of embedded system technology, interface (second edition). Tsinghua university press.

[5] Lu ruiwen. Automatic identification technology. Beijing: chemical industry press. 2005 .

[6] Li qunfang, zhang zhijun. Single chip microcomputer principle and interface technology. Beijing: electronic industry press, 2002

[7] Lang weimin. Principle and application of radio frequency identification (RFID) technology. Beijing: mechanical industry publishing house, 2006

[8] Zhou yuanjun. Electronic tags (RFID) technology application in animal production and management [journal]. Animal husbandry and veterinary, heilongjiang province, 2007 (8) : 57-58

[9] Liu Kai. Smart meters data acquisition system design based on ZigBee protocol. Automation

[10] Xunfei hkust. XF - S4240 speech synthesis module data manual. Anhui: anhui university xunfei information technology co., LTD., 2007

[11] Wang renhua. Depicting the recent research progress and application of speech synthesis technology outlook [journal]. Zte communications, 2003, 9 (5) : 37-39. 